\title{
Complexes of $\mathrm{Li}, \mathrm{Na}$ and $\mathrm{Mg}$ with 2D Allotropies of Second and Third Period. A Theoretical Study
}

\section{Wilmer Esteban Vallejo Narváez}

Universidad Nacional Autónoma de México: Universidad Nacional Autonoma de Mexico

\section{Cesar Gabriel Vera de la Garza}

Universidad Nacional Autónoma de México: Universidad Nacional Autonoma de Mexico

\section{Luis Daniel Solís Rodríguez}

Universidad Nacional Autonoma de Mexico

\section{Serguei Fomine ( $\nabla$ fomine@unam.mx )}

Universidad Nacional Autonoma de Mexico Instituto de Investigaciones en Materiales https://orcid.org/0000-0002-7068-7579

\section{Research Article}

Keywords: nanoflakes, inorganic analogues of haeckelites, lithium, sodium and magnesium, DFT

Posted Date: October 21st, 2021

DOI: https://doi.org/10.21203/rs.3.rs-972983/v1

License: (1) (1) This work is licensed under a Creative Commons Attribution 4.0 International License. Read Full License

Version of Record: A version of this preprint was published at Journal of Molecular Modeling on December 30th, 2021. See the published version at https://doi.org/10.1007/s00894-021-05019-1. 
Corresponding author's contact details

\author{
Name: Serguei Fomine \\ Email:fomine@unam.mx \\ Institute for materials research, National Autonomous University of Mexico, Apartado Postal 70-360, \\ CU, Coyoacán, México DF 04510, México \\ Phone +525542494945
}




\title{
Complexes of $\mathrm{Li}, \mathrm{Na}$ and $\mathrm{Mg}$ with 2D allotropies of second and third period.
} A theoretical study

\author{
Wilmer Esteban Vallejo Narváez, Cesar Gabriel Vera de la Garza, Luis Daniel Solís Rodríguez and \\ Serguei Fomine* \\ Instituto de Investigaciones en Materiales, Universidad Nacional Autónoma de México, Apartado \\ Postal 70-360, CU, Coyoacán, México DF 04510, México.
}

Keywords: nanoflakes; inorganic analogues of haeckelites; lithium, sodium and magnesium; DFT.

\begin{abstract}
Complexes of $\mathrm{Li}, \mathrm{Na}$ and $\mathrm{Mg}$ with graphene, silicene, phosphorene nanoflakes (NFs) and their 2D allotropies have been studied at dispersion corrected TPSS/def-TZVP level of theory. The energy partition analysis of the complexes revealed that for most of the complexes exchange and correlation energies represent dominant contributions to the binding with strong charge transfer from metal atom to a NF. The exceptions are Mg complexes of graphene and phosphorene NFs where binding is due to dispersion and correlation terms. This difference is also reflected in large Mg-NF distances suggesting weak intermolecular interactions in these complexes. The calculated activation energies for metal hopping are easily achievable at room temperatures for carbon and silicon allotropies. However, they are significantly higher for phosphorus allotropies reaching almost $18 \mathrm{kcal} / \mathrm{mol}$. There is a reasonably good correlation between the activation energies for hopping and binding energies for graphene, silicene and phosphorene NFs. Such correlation is not observed however for graphene, silicene and phosphorene 2D allotropies.
\end{abstract}

\section{Introduction}

Although 2D materials have become a mainstream in materials chemistry, only after graphene discovery in 2004, [1] the first 2D material, molybdenum disulfide was obtained and studied, back to 1986.[2] 2D materials fill the gap between 1D and 3D materials possessing valuable properties for applications especially in micro- and optoelectronics.[3] Graphene discovery pushed forward an extensive search for 2D allotropies of other elements. Following the graphene discovery, 2D 
allotropies of boron, phosphorus, silicon and germanium have been obtained too.[4-9] Although all 2D allotropies share the same topology, possessing honeycomb structure, their physical structural and chemical properties are very different. Thus, graphene and borophene are plane due to $s p^{2}$ hybridization common for $2^{\text {nd }}$ row elements, while $\mathrm{Si}, \mathrm{P}$ and Ge honeycomb allotropies have buckled geometry. The electronic properties of the allotropies change too from metallic for borophene to semiconductor for phosphorene. Although honeycomb allotropies are the most stable 2D allotropies for all these elements, they are not the only possible ones. Thus, carbon allotropies consisting of fused azulene rings known as "haeckelites" [10] (Fig. 1) are dynamically stable and occur naturally as Stone -Wales defects in graphene.[11] Moreover, it has been recently demonstrated theoretically, that silicon, phosphorus and germanium, but not boron are also capable of forming stable 2D allotropies of "haeckelite" topology.[12] Moreover, inorganic haeckelites were found to be relatively more stable than honeycomb structures compared to the corresponding carbon counterparts. It has been found that haeckelites have much higher affinity to Li atoms than graphene which make them promising anode materials for Li-ion batteries.[13] Although Li-ion [14] batteries have second highest possible theoretical energy densities after proton batteries, lithium is expensive and chemically very active. Possible alternative to $\mathrm{Li}$ - ion batteries are $\mathrm{Na}$ - and $\mathrm{Mg}$-ion batteries. $[15,16]$ Sodium is much cheaper than $\mathrm{Li}$, although more chemically active and offering lower theoretical energy density. Magnesium, on the other hand is not only much more accessible than $\mathrm{Li}$, it also more environmentally stable and offers higher energy density than Na. Haeckelites, including new inorganic systems are potentially interesting anode materials for Li-ion, Na-ion and Mg-ion batteries, therefore, the understanding of the interaction nature between 2D systems and metal atoms is of great importance. The aim of this manuscript is to study the interaction nature of $\mathrm{Li}, \mathrm{Na}$ and $\mathrm{Mg}$ atoms with organic and inorganic haeckelites and to compare the results with these for honeycomb allotropies. Moreover, the mobility of metal atoms along 2D surfaces has also been explored.

\section{Computational details}

2D allotropies were modeled as passivated by hydrogen atoms nanoflakes (NFs) containing 96 heavy atoms (Fig. 2) All calculations were carried out using gradient corrected meta TPSS functional in combination with triple zeta quality def-TZVP basis set.[17, 18] Grimm D3bj dispersion correction was applied in all cases.[19] TPSS functional offers a reasonable tradeoff between speed and 
accuracy.[17] Frequency calculations were run for all structures to ensure that minimum or transition state was found. All calculations were carried out using TURBOMOLE 7.5 code.[20] To obtain deeper insight into the interaction nature between a NF and metal atom, energy decomposition analysis (EDA) was used.[21] The total interaction energy ( $E_{\text {int }}$ ) was partitioned in physically meaningful contributions.

$\mathrm{E}_{\text {int }}=\mathrm{E}_{\mathrm{el}}+\mathrm{E}_{\mathrm{ex}}+\mathrm{E}_{\mathrm{orb}}+\mathrm{E}_{\mathrm{corr}}+\mathrm{E}_{\text {disp }}$

where $E_{e l}$ is electrostatic interaction, $E_{e x}$ is exchange interaction, $E_{o r b}$ is the orbital relaxation energy, $E_{c o r r}$ is the correlation energy and $E_{\text {disp }}$ is the dispersion energy. In our analysis we neglected $E_{\text {orb }}$ term since in all cases it was smaller than $0.001 \mathrm{kcal} / \mathrm{mol}$.

Metallic complexes with honeycomb NFs are denoted as $\mathbf{X}-\mathbf{M e}$, where $\mathbf{X}$ stands for the element symbol of the NF and Me - for metal atom type. In case of haeckelite allotropies, the metal complexes are marked as HX5-Me or HX7-Me where $\mathbf{X}$ stands for the element, $\mathbf{M e}-$ for the metal atom type and $\mathbf{5}$ or $\mathbf{7}$ is the complexation site: pentagon or heptagon.

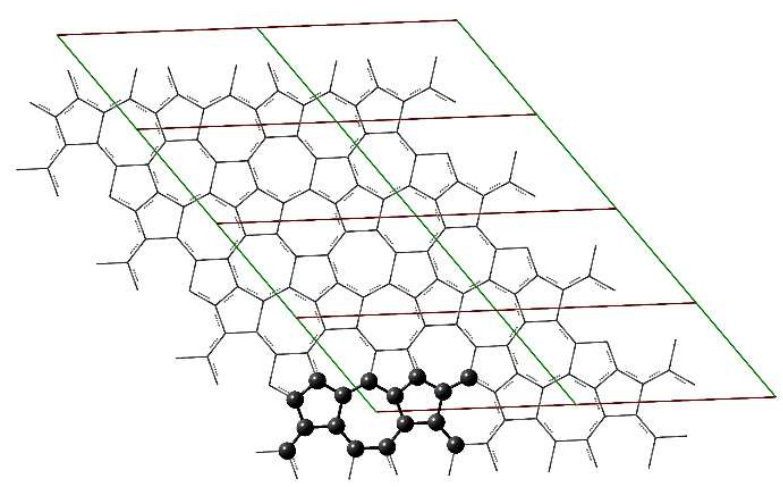

Fig. 1 Haeckelite topology. 


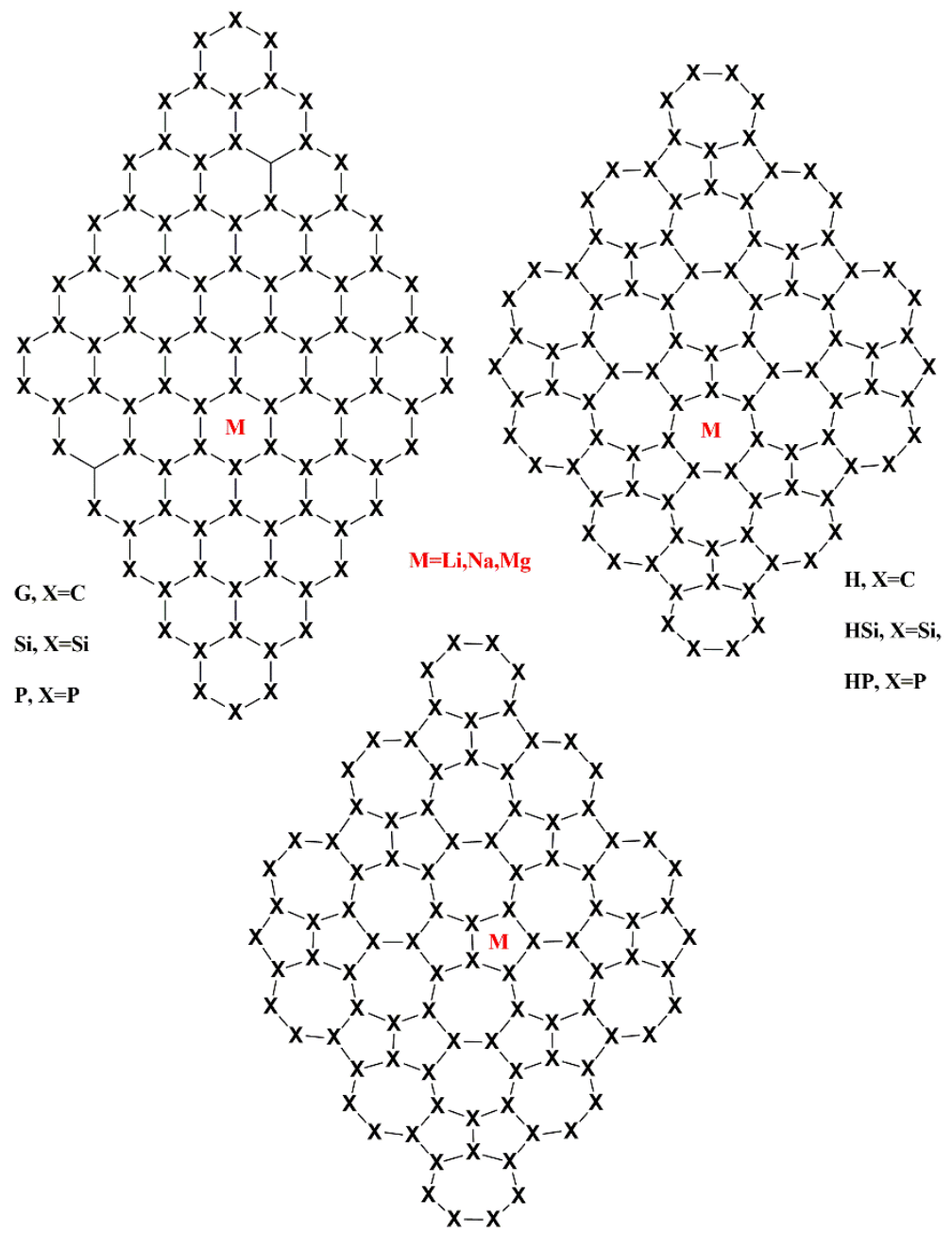

Fig.2 Topologies of studied NF complexes with Li, Na and Mg.

\section{Results and Discussion.}

\section{Geometry}

Fig. 2 depictures the topology of studied metal complexes. In almost all cases the metal atom is in the center and slightly above the polygon. The exception is HSi5 complexes where in stable structure the metal atom interacts with two pentagons at once. Corresponding optimized geometries are shown in the Fig.3. Optimized Cartesian coordinates of the complexes are in supporting information. 


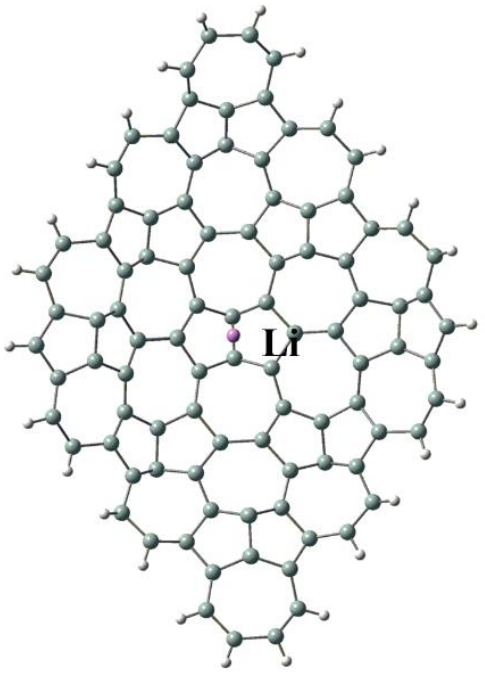

HSi5-Li

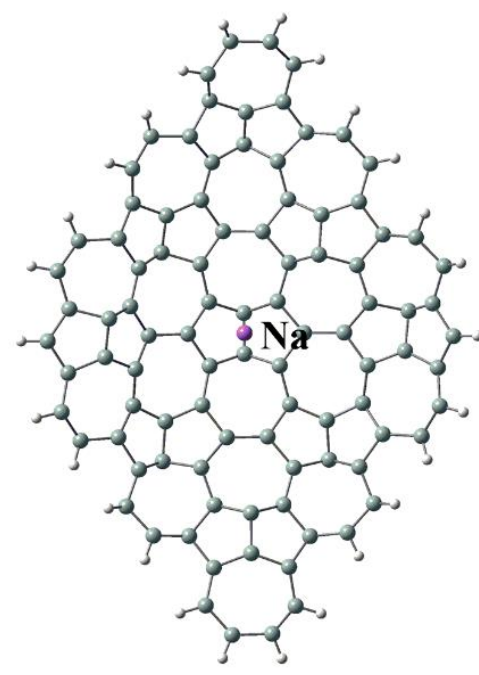

HSi5-Na

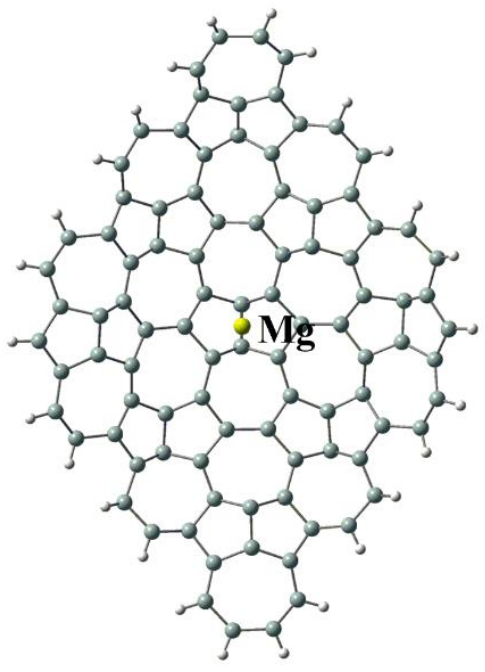

HSi5-Mg

Fig.3. Optimized geometries of HSi5-Me complexes

Fig.4 shows shortest distances in complexes between the metal and the NF atoms. For Li and $\mathrm{Na}$ there is a clear correlation of shortest distances with both valence radii of metal atoms and these of NF atoms. Thus, the largest distances are detected for Si-Na, HSi5-Na and HSi7-Na complexes in accordance with valence radii of $\mathrm{Na}$ and $\mathrm{Si}$ which are the biggest among the metal atoms and the NF atoms, respectively. However, complexes with magnesium do not follow this trend, especially in the case of G-Mg complex with the longest distance out of all complexes. We will discuss this exception latter. Studied NFs have 5-, 6- and 7- member rings as complexation sites and the complexation preference depends on the metal atom size and the complex shape. For nearly plane $\mathbf{H}$ and $\mathbf{S i}$ systems, metal-NF distance in complexes increases with the atom size. The situation becomes more complex for HSi, P and HP NFs which are not plane, the correlation between the atom size and the atom distance is lost for these complexes. The analysis of the interaction nature is helpful for these cases. 


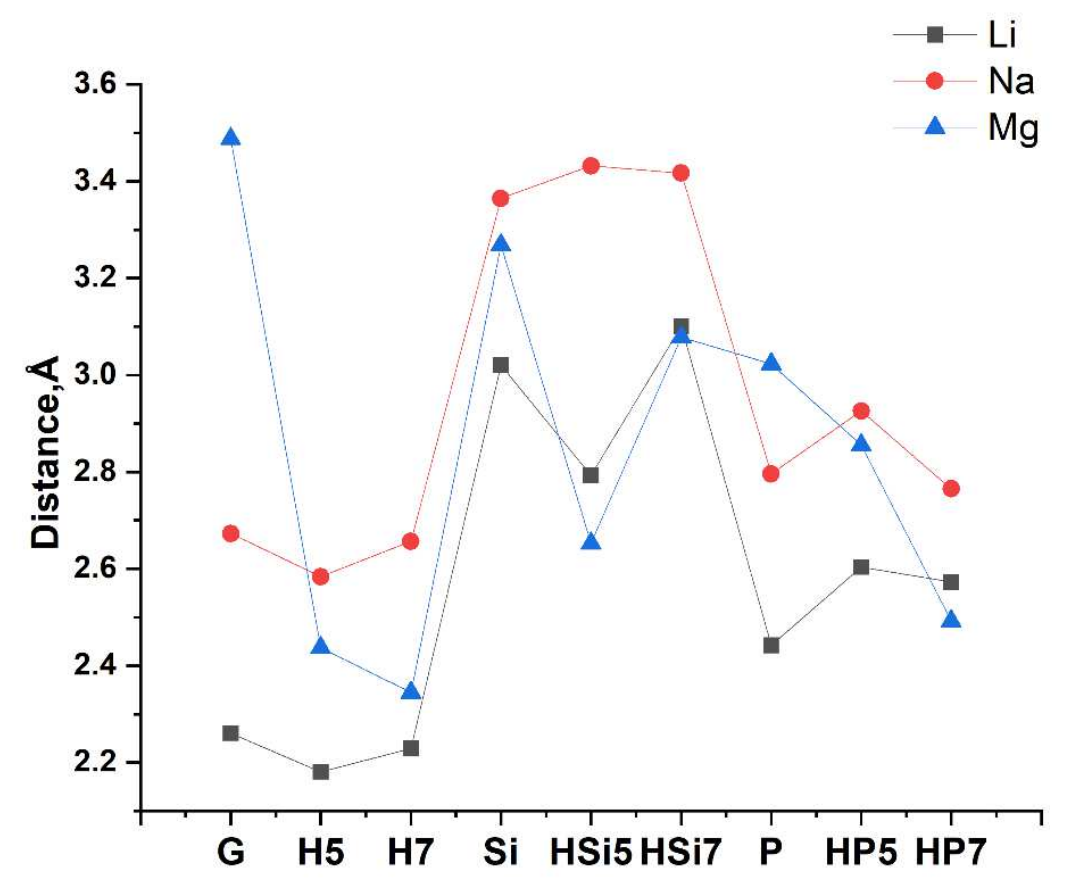

Fig.4 Shortest distances between metal and NF atoms in metal complexes.

\section{Interaction energies}

Fig.5, 6, 7, 8 and 9 show the total binding energies and their components for studied complexes: electrostatic, exchange, correlation and dispersion, respectively. As seen from the Fig.5, for all complexes Mg shows the lowest binding energies except for HP7-Mg system, while Li binding energies are the highest. Graphene shows smallest affinity to all types of metal atoms while the binding energies for silicene and phosphorene or for their allotropies are notably higher. The lowest binding energy is detected for G-Mg complexes. Inspecting the different terms to the binding energy for G-Mg complex, it was found that the dispersion and correlation are the most important binding contributions, electrostatic and exchange interaction are very low in comparison to other systems suggesting mostly weak intermolecular interaction in this complex. Natural charge of $\mathrm{Mg}$ atom is essentially 0 (Fig.10) and electronic configuration of $\mathrm{Mg}$ is $3 \mathrm{~S}^{2}$, similar to that in $\mathrm{Mg}$ atom. On the other hand, haeckelite $\mathbf{H}$ shows much better affinity to $\mathbf{M g}$ atom, the most important binding contributions comes from exchange and correlation interactions suggesting chemical bonding between $\mathbf{H}$ and $\mathbf{M g}$, dispersion becomes less important in this case. $\mathbf{M g}$ natural chargee in $\mathbf{H 5}-\mathbf{M g}$ 
and H7-Mg complexes are between 0.9 and 1.0 while natural electron configuration of $\mathrm{Mg}$ atom becomes $3 \mathrm{~S}^{1}$, implying total electron transfer from $\mathrm{Mg}$ to haeckelite. Such a big difference between two isomeric carbon NFs is due to higher aromatic stabilization of benzene fragments of graphene compared to haeckelites resulting in lower electron affinity of graphene NF compared to haeckelite ones. [12] In case of $\mathrm{Li}$ and $\mathrm{Na}$ there is a complete electron transfer from metal atom to the NF for all complexes. For haeckelite carbon allotropies the binding energies of $\mathbf{H}$ complexes are higher compared to graphene. The difference is due to lower electrostatic repulsion and more negative exchange interactions in case of haeckelite. Correlation and dispersion barely affect resulting binding energies. Similar trends for binding energies can be observed for silicon allotropies, however, binding energies are higher compared to carbon counterparts and increase in the order $\mathrm{Mg}<\mathrm{Na}<\mathrm{Li}$. Higher binding energies for silicone haeckelite allotropy compared to silicene are due to better relation between electrostatic repulsion, exchange attraction and better correlation stabilization for the formers. Dispersion does not play important role there. The electrostatic repulsion in all silicone containing NF-metal complexes decreases in the order $\mathrm{Mg}>\mathrm{Na}>\mathrm{Li}$, thus explaining the relative stability of the complexes for different metals. Other contributions are less important. It is interesting to note (Fig.10), that for Mg complexes of silicone haeckelite allotropy and especially for HSi5-Mg one, the natural charge of $\mathrm{Mg}$ atom is 1.4 - the second highest out of all complexes which corresponds to natural electron configuration of $\mathrm{Mg}$ atom $35^{0.56}$. In case of $\mathbf{S i}-\mathbf{M g}$ complex, the natural charge at $\mathrm{Mg}$ atom is only 0.7 . This is due to almost $1 \mathrm{eV}$ higher electron affinity of HSi compared to silicene.[12]

Binding energy for complexes with phosphorene NFs and phosphorene allotropy follows the trend found for other complexes Li> Na> Mg for all except for HP7-Me systems where the highest binding energy was found for $\mathrm{Mg}$, while $\mathrm{Li}$ and $\mathrm{Na}$ have almost the same binding energies. This anomaly can be explained analyzing charges and geometry. As seen the shortest $\mathbf{M g}-\mathbf{P}$ bond is about $0.5 \AA$ shorter in HP7-Mg compared to P-Mg and HP5-Mg, and the second shortest Mg-P distance in HP7-Mg is $2.54 \AA$, still $0.4 \AA$ A shorter compared to the shortest Mg-P distance in P-Mg and HP5-Mg complexes. This suggests that in HP7-Mg complex Mg atom forms two polar bond with different $\mathrm{P}$ atoms which is further conformed by high positive natural charge of 1.4 at $\mathrm{Mg}$, compared to 0.52 and 0.63 in PMg and HP5-Mg, respectively. Natural electron configuration of $\mathrm{Mg}$ in HP7-Mg is $3 S^{0.54}$, showing that two valence electrons of $\mathrm{Mg}$ participate in the formation of chemical bonding resulting in highest binding energy out of all complexes. Large seven membered ring in HP promotes interaction of $\mathrm{Mg}$ atom with two phosphorus at the same time. EDA shows that for phosphorus containing NFs, 
$\mathrm{Li}$ and $\mathrm{Na}$ have similar electrostatic repulsion components, exchange and correlation energies. Dispersion interaction is higher for $\mathrm{Na}$ due to higher polarizability compared to Li. The most important binding contributions come from exchange and correlation energies. $\mathrm{Mg}$ complexes are different. The contribution to the binding energy depends on the complex type. For P-Mg complex the most important binding terms are correlation and dispersion, indication of mostly intermolecular interactions, while for HP5-Mg and HP7-Mg exchange and correlation attraction dominate in the binding. Considering the most important binding terms and distances between $\mathrm{Mg}$ and $\mathrm{P}$ atoms in P-Mg, HP5-Mg and HP7-Mg complexes it can be concluded that unlike HP5-Mg and HP7-Mg, where there is a covalent interaction between the NF and $\mathbf{M g}$ atom, in $\mathbf{P}-\mathbf{M g}$ these interactions are mostly intermolecular. In P-Mg complex the contributions from electrostatic and exchange interactions are very small compared to HP5-Mg and HP7-Mg while P-Mg distance in P$\mathrm{Mg}$ is large, larger than the sum of covalent radii of $\mathrm{Mg}$ and $\mathrm{P}$.

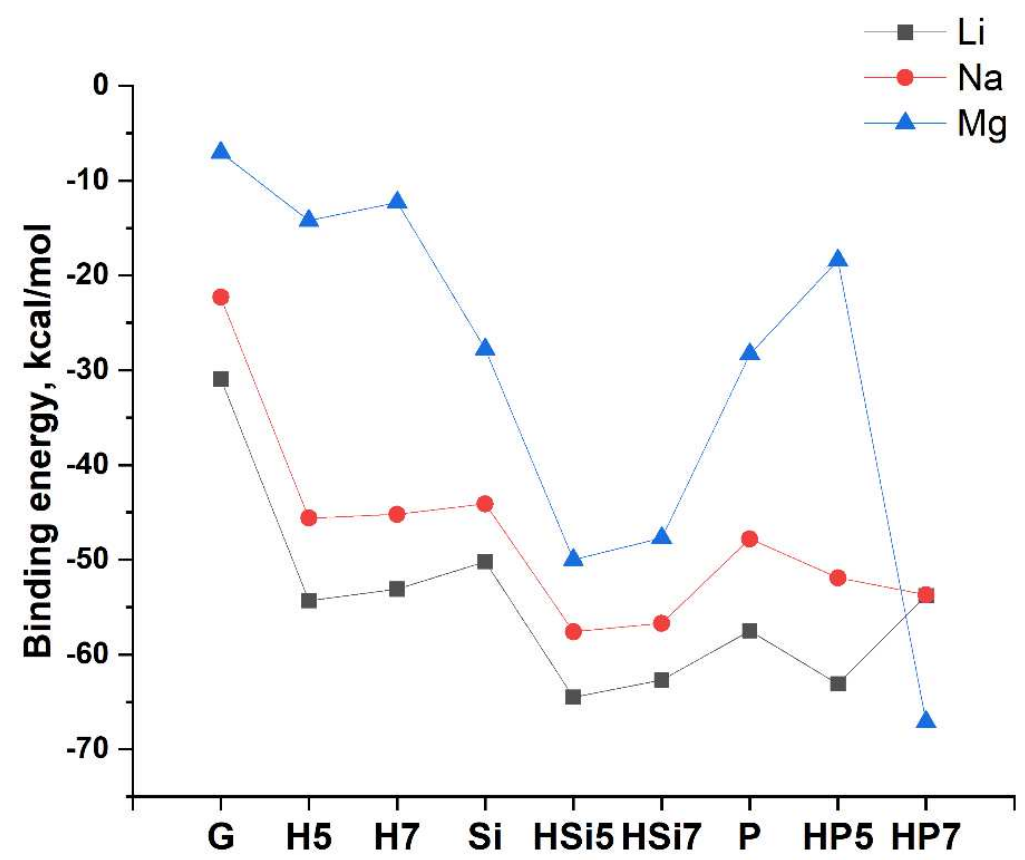

Fig.5 Total binding energies in studied complexes. 


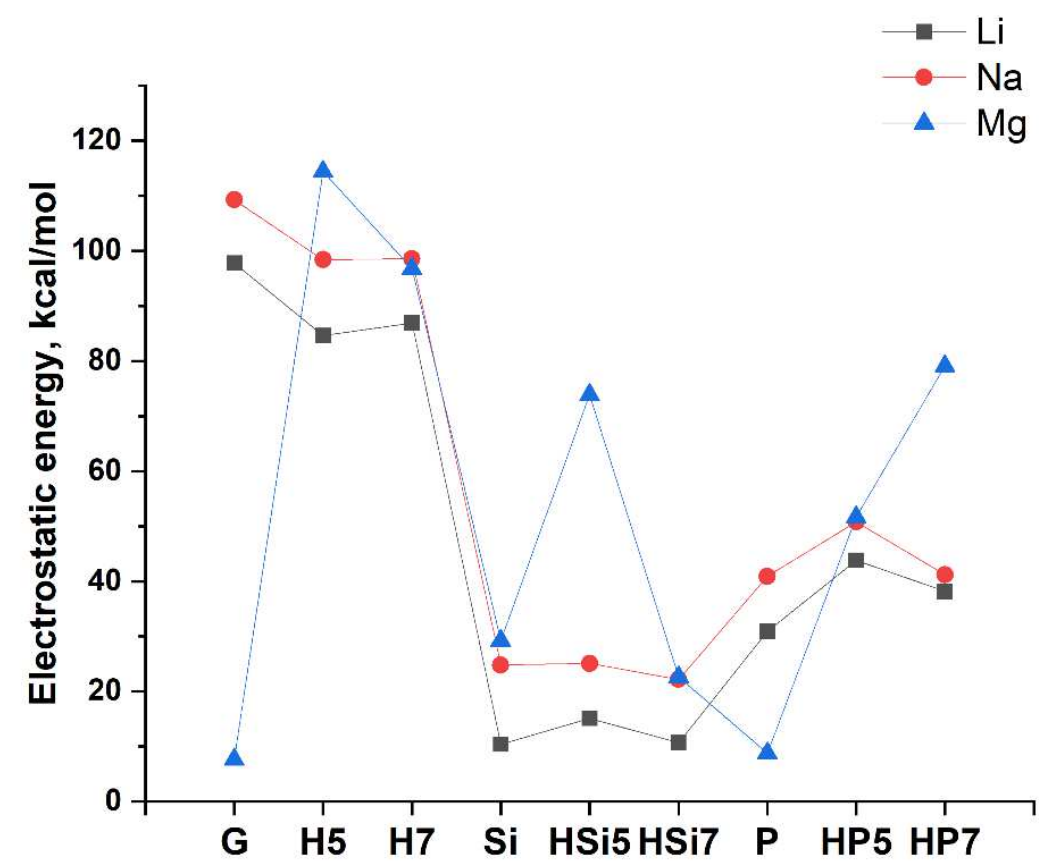

Fig.6 Electrostatic interaction in studied complexes.

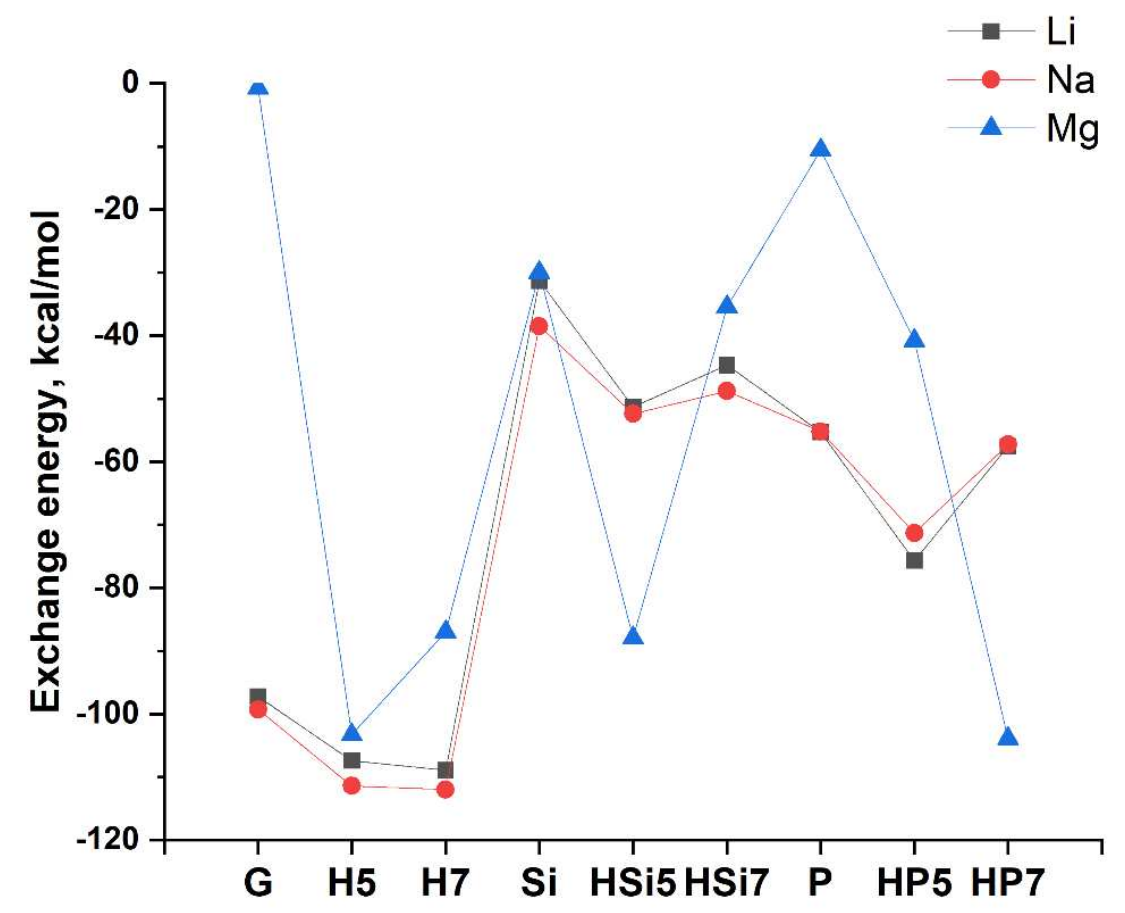


Fig.7 Exchange interaction in studied complexes.

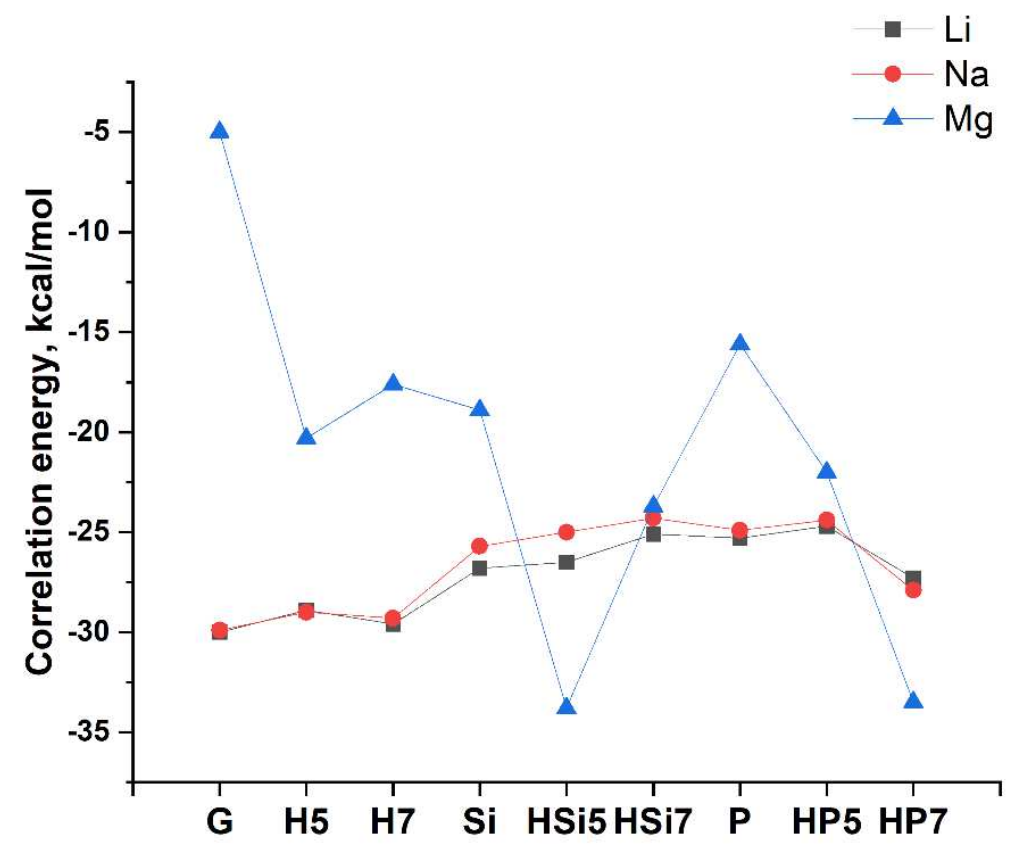

Fig.8 Correlation interaction in studied complexes.

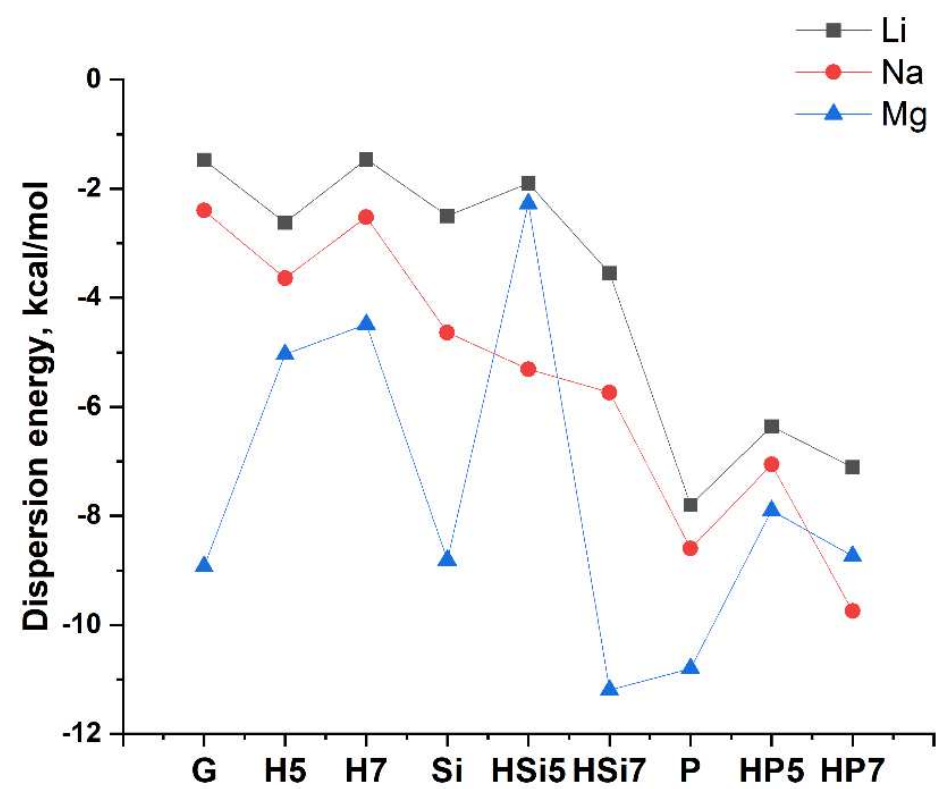

Fig. 9 Dispersion interaction in studied complexes 


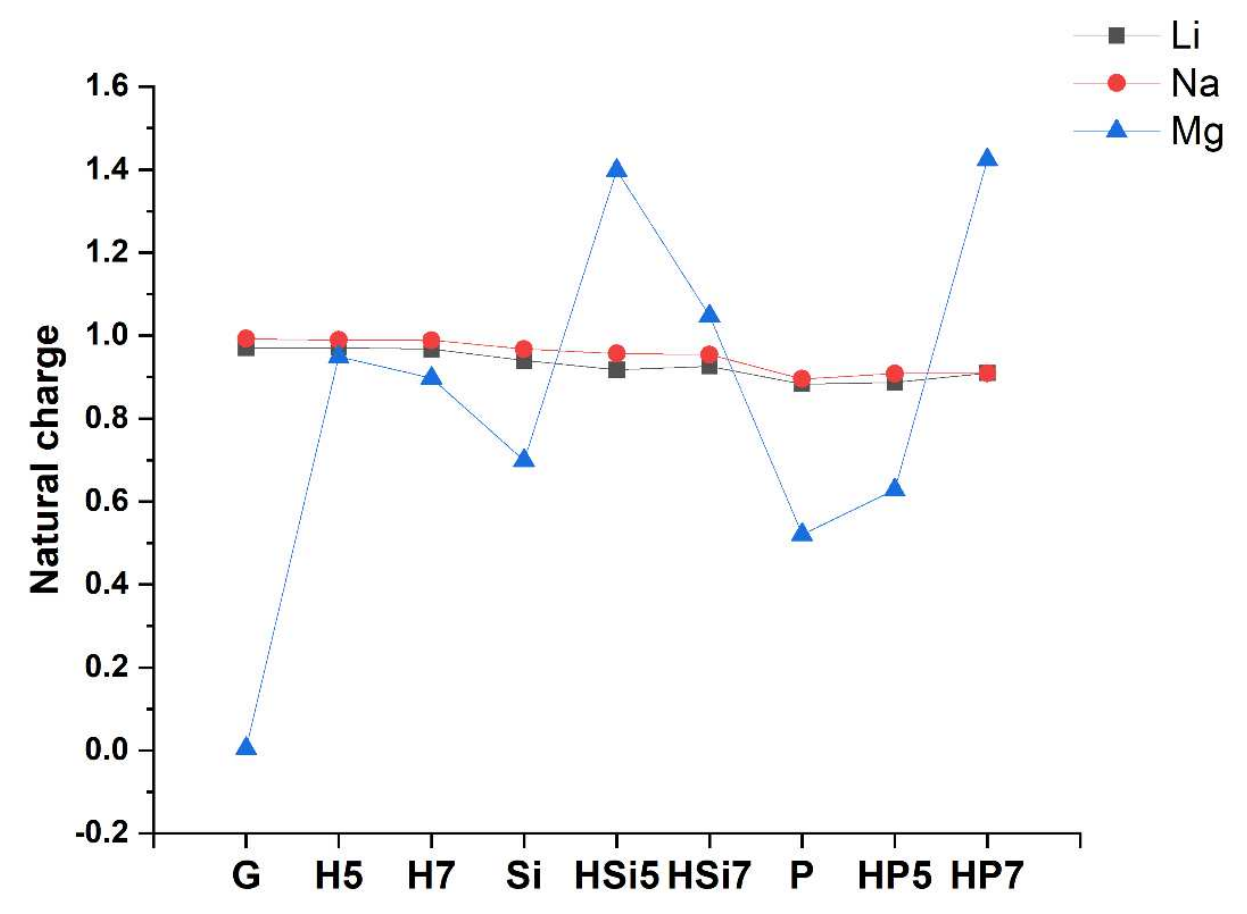

Fig. 10 Natural charges of metal atoms in studied complexes

\section{Activation energies for hopping}

Fig. 11 and 12 show calculated activation energies for metal atoms diffusion along the NFs. In case of hexagonal allotropies: graphene, silicene and phosporene, (Fig.11) the activation energies for hopping increase in this direction being the lowest for graphene and highest for phosphorene. As seen from the Fig 13, there is a certain correlation between the binding and activation energies with R-squared of $\mathbf{0 . 3 8}$. The highest activation energies correspond to P-Li, having the highest binding energy. The lowest activation energy was calculated for $\mathbf{G}-\mathbf{M g}$, this complex shows the lowest binding energy too. The highest activation energies are for $\mathrm{Li}$ while the smallest are for $\mathrm{Mg}$, except for phosphorene where the lowest activation energy is for $\mathrm{Na}$. 


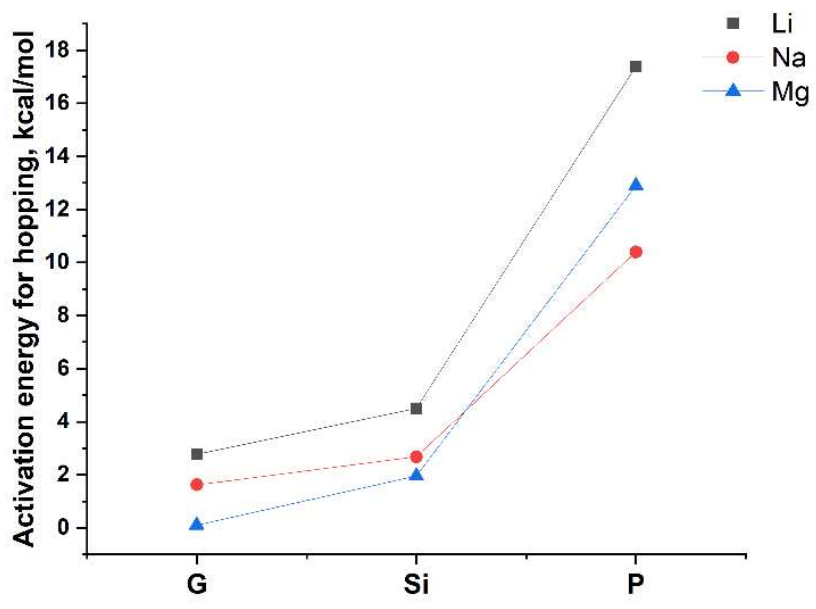

Fig.11 Activation energies for metal hopping in complexes of graphene (G), silicene (Si) and phosphorene (P)

In case of haeckelite allotropies of carbon, silicon and phosphorus there are 3 different ways of hopping for metal atoms: heptagon-heptagon (7-7), pentagon-pentagon (5-5) and heptagonpentagon (7-5 or 5-7). 5-5 Transitions states (TSs) were detected only for Li atom and only for carbon and phosphorus allotropies. For other metals and systems, the structure of TS corresponds to a minimum. This is related to the larger sizes of $\mathrm{Na}$ and $\mathrm{Mg}$ atoms compared to $\mathrm{Li}$ and small size of pentagon ring where $\mathrm{Na}$ and $\mathrm{Mg}$ atoms can form a complex with two pentagons at the same time as shown in Fig.3. Hopping activation energies for carbon and silicon allotropies are close to these of graphene and silicene being in the range between 0.2 and $6 \mathrm{kcal} / \mathrm{mol}$, all easily assessable at room temperature. The activation energies vary greatly for HP system from almost $17 \mathrm{kcal} / \mathrm{mol}$ for $\mathrm{Na}(5-7)$ to $0.5 \mathrm{kcal} / \mathrm{mol}$ for Li (5-5), however they are generally lower than these estimated for phosphorene.

The estimation of average activation energies of metal atoms among all studied NF type, Mg shows the lowest average activation energy of $4.15 \mathrm{kcal} / \mathrm{mol}, \mathrm{Na}-5.70$ and $\mathrm{Li}-$ the highest $6.13 \mathrm{kcal} / \mathrm{mol}$. 


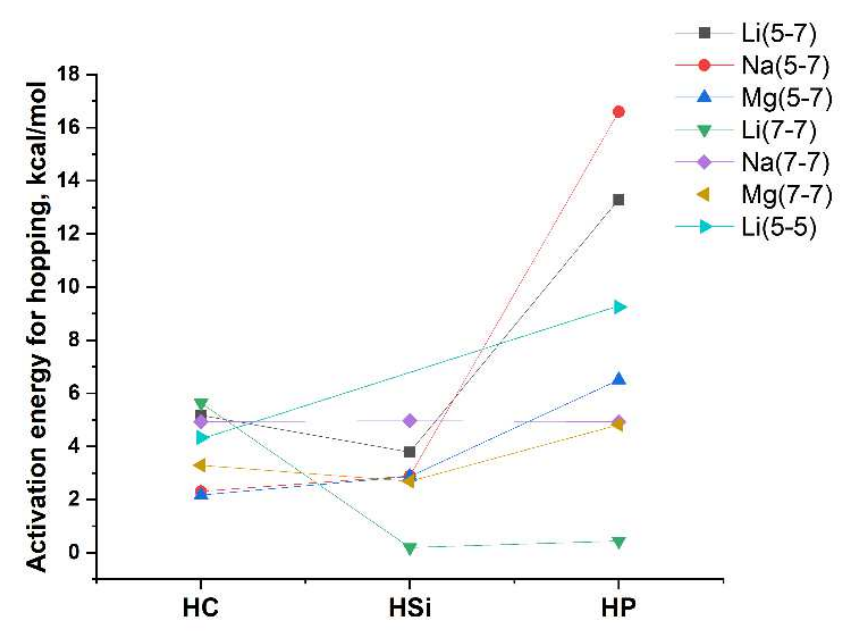

Fig.12 Activation energies for metal hopping in complexes of $\mathbf{H}, \mathbf{H S i}$ and $\mathbf{H P}$ allotropies.

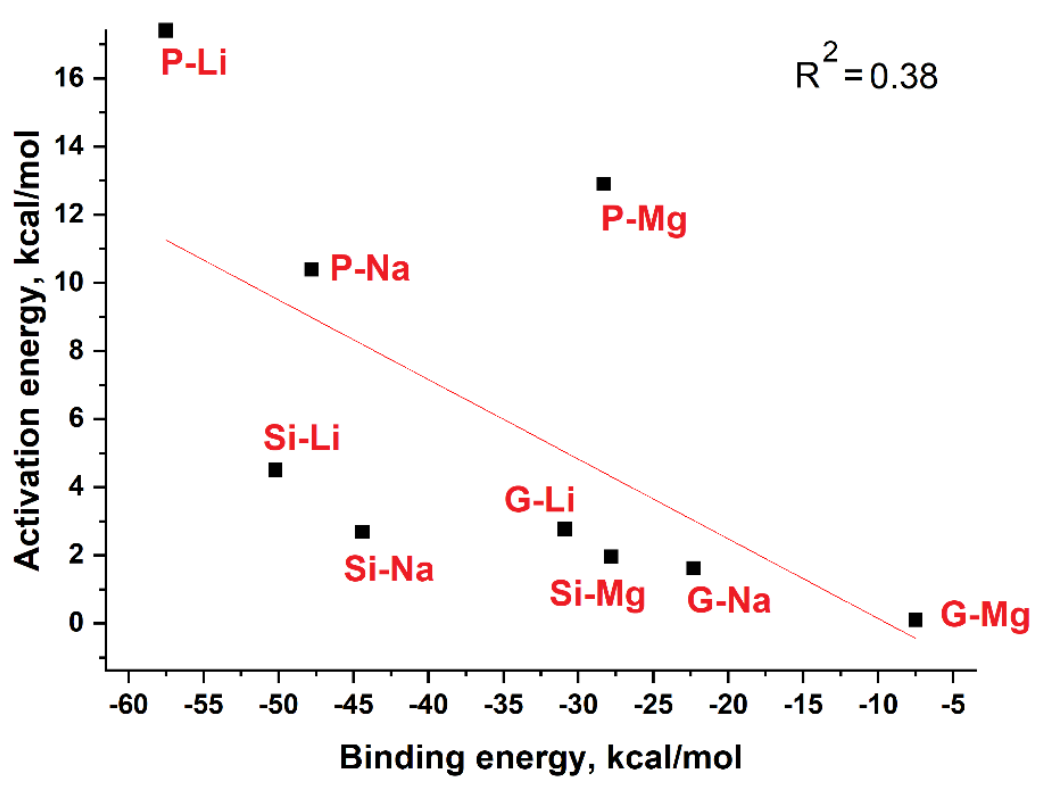

Fig 13 Binding vs activation energies of metal hopping for graphene, silicene and phosphorene

NFs.

As can be seen, the average activation energies of metal atom migrations do not vary much with metal nature, however, the average activation energies for different NF types do vary (Fig 14). The lowest average activation energies were calculated for graphene NFs, while the highest one was detected for phosphorene. The average activation energies for hackelites and its silicon analogue 
differ by only $2 \mathrm{kcal} / \mathrm{mol}$ from these for graphene and silicene, while HP allotropy shows noticeably lower hopping activation energies compared to phosphorene. A combination of graphene and $\mathrm{Mg}$ leads to the lowest activation energy of hopping while P-Li have the highest activation energy.

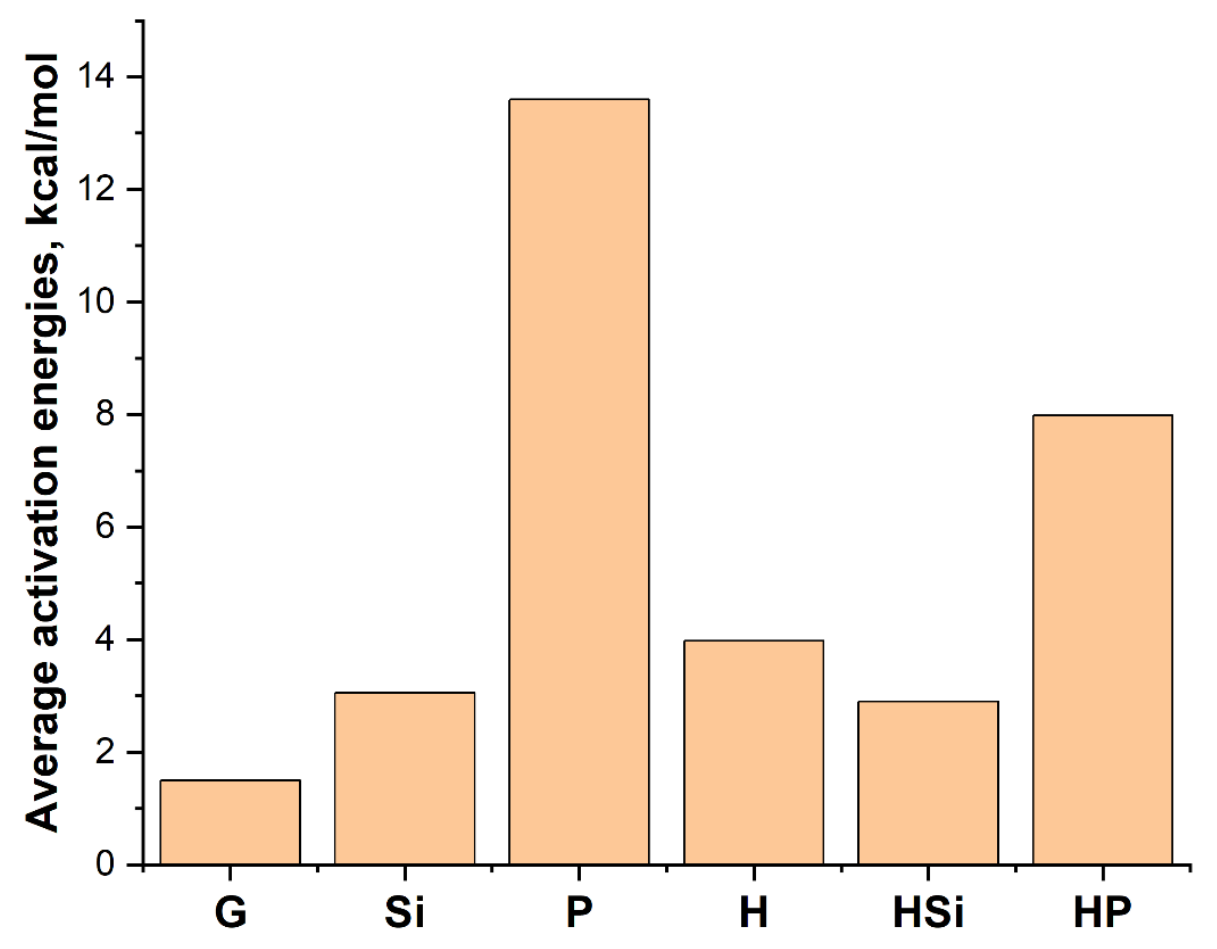

Fig. 14 Average activation energies for hopping for different NF types for all studied metals.

\section{Conclusions}

Complexes formed by $\mathrm{Mg}$ are quite different from these formed by $\mathrm{Na}$ and $\mathrm{Li}$. In the latter case there is always a complete electron transfer from metal atom to a NF and the most important contribution to the binding energy comes from exchange and correlation stabilization. Binding of $\mathrm{Mg}$ to a NF depends strongly on the NF type, ranging from G-Mg complex where there is no charge transfer from $\mathrm{Mg}$ to NF with dispersion being the most important contribution to binding, to $\mathbf{H P 7 - M g}$ complex showing the highest binding energy out of all studied complexes where Mg transfers almost 1.5 electrons to the NF. In this case the most important contributions to the binding are from 
exchange and correlation. The Mg - NF distance is 1 A shorter for the latter complex. Generally, the binding energies increase in the order $\mathrm{Mg}<\mathrm{Na}<\mathrm{Li}$ for all types of $\mathrm{NFs}$ with exchange and correlation contributing to binding, being the most important. Dispersion is only important for $\mathbf{G}-\mathbf{M g}$ complex. Haeckelite allotropies for carbon and silicon show higher binding energies compared to graphene and silicene, unlike phosphorus allotropies where there is no clear difference between phosphorene and HP allotropy which could be related to weak $p$ electron coupling between phosphorus atoms in these systems. The activation energies for hopping are easily achievable at room temperatures for carbon and silicon allotropies. However, they are significantly higher for phosphorene and HP allotropies achieving almost $18 \mathrm{kcal} / \mathrm{mol}$. There is a reasonably good correlation between the activation and binding energies for graphene, silicene and phosphorene NFs. Such correlation is not observed however for HC, HSi and HP allotropies.

\section{Acknowledgments}

We acknowledge the financial support from PAPIIT (IN201219/31) and the financial support from CONACYT (Grant 251684). W.E.V.N. acknowledges support from DGAPA of the UNAM under postdoctoral fellowship Grant No. CIIC/CTIC/4732/2O2O.

\section{Declarations}

\section{Finding}

PAPIIT (IN201219/31)

CONACYT (Grant 251684

DGAPA of the UNAM under postdoctoral fellowship Grant No. CJIC/CTIC/4732/202O.

\section{Conflicts of interest/Competing interests.}

We declare no conflicts of interests.

\section{Availability of data and material}


Not applicable

Code availability

TURBOMOLE V7.5 2020, a development of University of Karlsruhe and Forschungszentrum Karlsruhe GmbH, 1989-2007, TURBOMOLE GmbH, since 2007; available from https://www.turbomole.org

\section{Authors' contributions}

Cesar Gabriel Vera de la Garza - a PhD student - performed EDA analysis

Wilmer Esteban Vallejo Narváez - a postdoctoral fellow - transition state search, and partial manuscript writing.

Luis Daniel Solís Rodríguez - an undergraduate student - routine optimizations of complexes

Seguei Fomine - group leader, the manuscript idea and writing. 


\section{References}

1. Novoselov KS, A K Geim, S V Morozov, et al (2004) Electric Field Effect in Atomically Thin Carbon Films. Science 306:666-669. https://doi.org/10.1126/science.1102896

2. Joensen P, Frindt RF, Morrison SR (1986) Single-layer MoS2. Materials Research Bulletin 21:457-461. https://doi.org/10.1016/0025-5408(86)90011-5

3. Chang H, Wu H (2013) Graphene-Based Nanomaterials: Synthesis, Properties, and Optical and Optoelectronic Applications. Advanced Functional Materials 23:. https://doi.org/10.1002/adfm.201202460

4. Aufray B, Kara A, Vizzini S, et al (2010) Graphene-like silicon nanoribbons on $\mathrm{Ag}(110)$ : $\mathrm{A}$ possible formation of silicene. Applied Physics Letters 96:.

https://doi.org/10.1063/1.3419932

5. Lalmi B, Oughaddou H, Enriquez H, et al (2010) Epitaxial growth of a silicene sheet. Applied Physics Letters 97:. https://doi.org/10.1063/1.3524215

6. Castellanos-Gomez A (2015) Black Phosphorus: Narrow Gap, Wide Applications. The Journal of Physical Chemistry Letters 6:. https://doi.org/10.1021/acs.jpclett.5b01686

7. Dávila ME, Xian L, Cahangirov S, et al (2014) Germanene: a novel two-dimensional germanium allotrope akin to graphene and silicene. New Journal of Physics 16:. https://doi.org/10.1088/1367-2630/16/9/095002

8. Mannix AJ, Zhou X-F, Kiraly B, et al (2015) Synthesis of borophenes: Anisotropic, twodimensional boron polymorphs. Science 350:. https://doi.org/10.1126/science.aad1080

9. Feng B, Zhang J, Zhong Q, et al (2016) Experimental realization of two-dimensional boron sheets. Nature Chemistry 8:. https://doi.org/10.1038/nchem.2491

10. Enyashin AN, Ivanovskii AL (2011) Graphene allotropes. physica status solidi (b) 248:18791883. https://doi.org/10.1002/pssb.201046583

11. Chuvilin A, Meyer JC, Algara-Siller G, Kaiser U (2009) From graphene constrictions to single carbon chains. New Journal of Physics 11:083019. https://doi.org/10.1088/13672630/11/8/083019

12. de la Garza CGV, Narváez WEV, Rodríguez LDS, Fomine S (2021) Novel 2D allotropic forms and nanoflakes of silicon, phosphorus, and germanium: a computational study. Journal of Molecular Modeling 27:142. https://doi.org/10.1007/s00894-021-04775-4

13. Mpourmpakis G, Froudakis GE, Tylianakis E (2006) Haeckelites: A promising anode material for lithium batteries application. An $a b$ initio and molecular dynamics theoretical study. Applied Physics Letters 89:. https://doi.org/10.1063/1.2403922

14. Xu Y, Wu X, Ji X (2021) The Renaissance of Proton Batteries. Small Structures 2:. https://doi.org/10.1002/sstr.202000113 
15. Slater MD, Kim D, Lee E, Johnson CS (2013) Sodium-Ion Batteries. Advanced Functional Materials 23:. https://doi.org/10.1002/adfm.201200691

16. Aurbach D, Lu Z, Schechter A, et al (2000) Prototype systems for rechargeable magnesium batteries. Nature 407:. https://doi.org/10.1038/35037553

17. Tao J, Perdew JP, Staroverov VN, Scuseria GE (2003) Climbing the Density Functional Ladder: Nonempirical Meta-Generalized Gradient Approximation Designed for Molecules and Solids. Physical Review Letters 91:. https://doi.org/10.1103/PhysRevLett.91.146401

18. Schäfer A, Huber C, Ahlrichs R (1994) Fully optimized contracted Gaussian basis sets of triple zeta valence quality for atoms Li to Kr. The Journal of Chemical Physics 100:. https://doi.org/10.1063/1.467146

19. Grimme S, Ehrlich S, Goerigk L (2011) Effect of the damping function in dispersion corrected density functional theory. Journal of Computational Chemistry 32:.

https://doi.org/10.1002/jcc.21759

20. TURBOMOLE V7.5 2020, a development of University of Karlsruhe and Forschungszentrum Karlsruhe GmbH, 1989-2007, TURBOMOLE GmbH, since 2007; available from https://www.turbomole.org.

21. Su P, Li H (2009) Energy decomposition analysis of covalent bonds and intermolecular interactions. The Journal of Chemical Physics 131:. https://doi.org/10.1063/1.3159673 